\title{
Concurrent AtC coupling based on a blend of the continuum stress and the atomistic force
}

\author{
Jacob Fish ${ }^{\text {a,* }}$, Mohan A. Nuggehally ${ }^{\text {a }}$, Mark S. Shephard ${ }^{a}$, Catalin R. Picu ${ }^{\text {a }}$, \\ Santiago Badia ${ }^{\mathrm{b}}$, Michael L. Parks ${ }^{\mathrm{b}}$, Max Gunzburger ${ }^{\mathrm{c}}$ \\ ${ }^{a}$ Scientific Computation Research Center, Rensselaer Polytechnic Institute, 110 8th Street, Troy, NY 12180, United States \\ ${ }^{\mathrm{b}}$ Computational Mathematics and Algorithms, MS-1320, Sandia National Laboratories, Albuquerque, NM 87185-1320, United States ${ }^{1}$ \\ ${ }^{\mathrm{c}}$ School of Computational Science, Florida State University, Tallahassee, FL 32306-4120, United States
}

Received 27 November 2006; received in revised form 21 May 2007; accepted 25 May 2007

Available online 10 June 2007

\begin{abstract}
A concurrent atomistic to continuum (AtC) coupling method is presented in this paper. The problem domain is decomposed into an atomistic sub-domain where fine scale features need to be resolved, a continuum sub-domain which can adequately describe the macroscale deformation and an overlap interphase sub-domain that has a blended description of the two. The problem is formulated in terms of equilibrium equations with a blending between the continuum stress and the atomistic force in the interphase. Coupling between the continuum and the atomistics is established by imposing constraints between the continuum solution and the atomistic solution over the interphase sub-domain in a weak sense. Specifically, in the examples considered here, the atomistic domain is modeled by the aluminum embedded atom method (EAM) inter-atomic potential developed by Ercolessi and Adams [F. Ercolessi, J.B. Adams, Interatomic potentials from first-principles calculations: the force-matching method, Europhys. Lett. 26 (1994) 583] and the continuum domain is a linear elastic model consistent with the EAM potential. The formulation is subjected to patch tests to demonstrate its ability to represent the constant strain modes and the rigid body modes. Numerical examples are illustrated with comparisons to reference atomistic solution. (c) 2007 Elsevier B.V. All rights reserved.
\end{abstract}

Keywords: Concurrent multiscale; Atomistic to continuum coupling; Overlap domain decomposition

\section{Introduction}

Engineers and scientists have realized the importance of analyzing all the relevant scales together and linking them properly for the problems that have features or phenomena of interest in different spatial and temporal scales. It has become crucial to understand the relationship of processes taking place across various length and time scales for the

\footnotetext{
${ }^{*}$ Corresponding author. Tel.: +15182766191.

E-mail addresses: fishj@rpi.edu (J. Fish),nuggehal@scorec.rpi.edu (M.A. Nuggehally), shephard@scorec.rpi.edu (M.S. Shephard), cpicu@, scorec.rpi.edu (C.R. Picu), sibadia@sandia.gov (S. Badia), mlparks@ sandia.gov (M.L. Parks), gunzburg@csit.fsu.edu (M. Gunzburger).

${ }^{1}$ Sandia is a multiprogram laboratory operated by Sandia Corporation, a Lockheed Martin Company, for the United States Department of Energy under contract DE-AC04-94-AL85000.
}

advancement of various fields like material science [22], pharmaceutical drugs and biology, micro and nano technology [26], etc. The macroscopic behavior of systems are predicted from continuum based theory and computational models, which traditionally have phenomenological constitutive relationships. But the macroscopic behavior is inherently governed by the physics taking place on multiple unresolved scales.

Multiscale modeling and simulation techniques can be broadly classified into two categories as sequential multiscale methods and concurrent multiscale methods. In sequential multiscale methods fine scale information is averaged and introduced into coarse scale models in the form of constitutive relations. In concurrent methods two or more models are simultaneously resolved in different regions of a problem domain. 
Macroscopic phenomena of interest such as fracture and fatigue of materials are a result of the physical processes occurring in the atomistic scale such as dislocations, voids and interstitials or even quantum scale processes such as reactions leading to corrosion. Disparity in the length scales between such coarse scale and fine scale phenomena can exceed $10^{10}$. It is prohibitive in terms of computational cost to model coarse scale phenomena from fine scale models alone. Often only localized areas of a vast problem domain need fine scale models to resolve the complicated fine scale processes while the rest of problem domain can be modeled with a coarse scale model. Concurrent multiscale methods are an effective tool to handle such situations. The focus here is on a concurrent method that couples a continuum model with an atomistic model.

Most of the work in concurrent modeling techniques is by coupling molecular statics or molecular dynamics with a continuum model. Following is a brief review of such concurrent modeling techniques available in the literature. Combined finite element and atomistic models to study crack propagation in crystals $[23,19]$ are some of the earliest works of atomistic/continuum coupling. A reference cited frequently is the macroscopic, atomistic, ab-initio

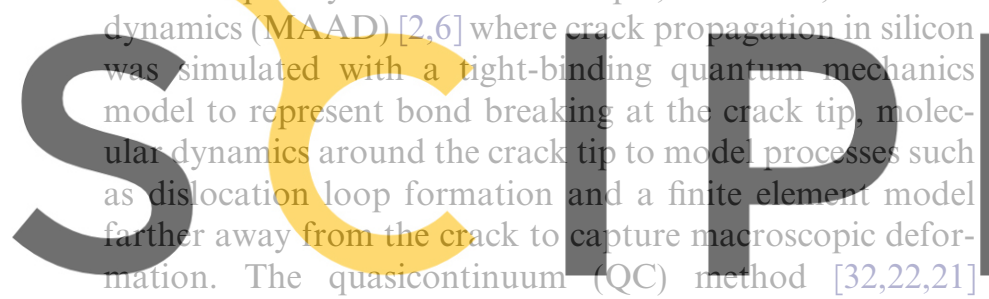
resolves the regions close to defects like dislocations, grain

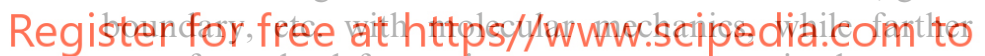
away from the defect region atoms are constrained to move in groups by the finite element shape functions and mesh, thereby greatly reducing the degrees of freedom in a problem. Finite-temperature quasicontinuum [12] is developed as a coarse-grained alternative to molecular dynamics for crystalline solids at constant temperature by using a combination of statistical mechanics and finite element interpolation functions. Coarse-grained molecular dynamics [27,28] uses a coarse graining procedure based on statistical mechanics to derive equations of motion for a finite element mesh from the equations of motion of molecular dynamics. The bridging domain method $[5,34]$ has been used to couple continuum to atomistics through an overlap region and study shock wave propagation from molecular region to the continuum region. Bridging scale method (BSM) has been adopted by Liu et al. $[33,24]$ where the solution is decomposed into fine scale and coarse scale parts and a projection operator is used to decouple the kinetic energy of the atomistic and the continuum subdomains. A concurrent multiscale approach based on multigrid principles was introduced in [15]. The Arlequin modeling technique [11] that has been proposed to locally modify a mathematical model to capture the required physics can be potentially used for concurrent multiscale problems.
A hybrid domain decomposition concurrent multiscale formulation is proposed in which two or more mathematical models with disparate scales (continuum and atomistic models specifically) coexist in different parts of a problem domain. The models interact through an overlap interphase sub-domain. The problem is formulated in terms of equilibrium equations for the whole problem domain; the atomistic force is blended with the continuum stress in the overlap interphase. Constraints are imposed between the degrees of freedom of the interacting models in the form of a weak compatibility in overlap interphase subdomains. Section 2 gives the details of the concurrent AtC coupling method. Patch tests are devised to test the correctness of the method, which is discussed in Section 3. Section 4 illustrates numerical examples. The last section gives a summary of the different sections. There are two distinguishing features of the proposed method. First, the problem is formulated in terms of an equilibrium equation that has a blend of the atomistic force and the continuum stress in the interphase. Second, coupling between the continuum and the atomistic models is through a weak compatibility of solution in an overlap interphase sub-domain.
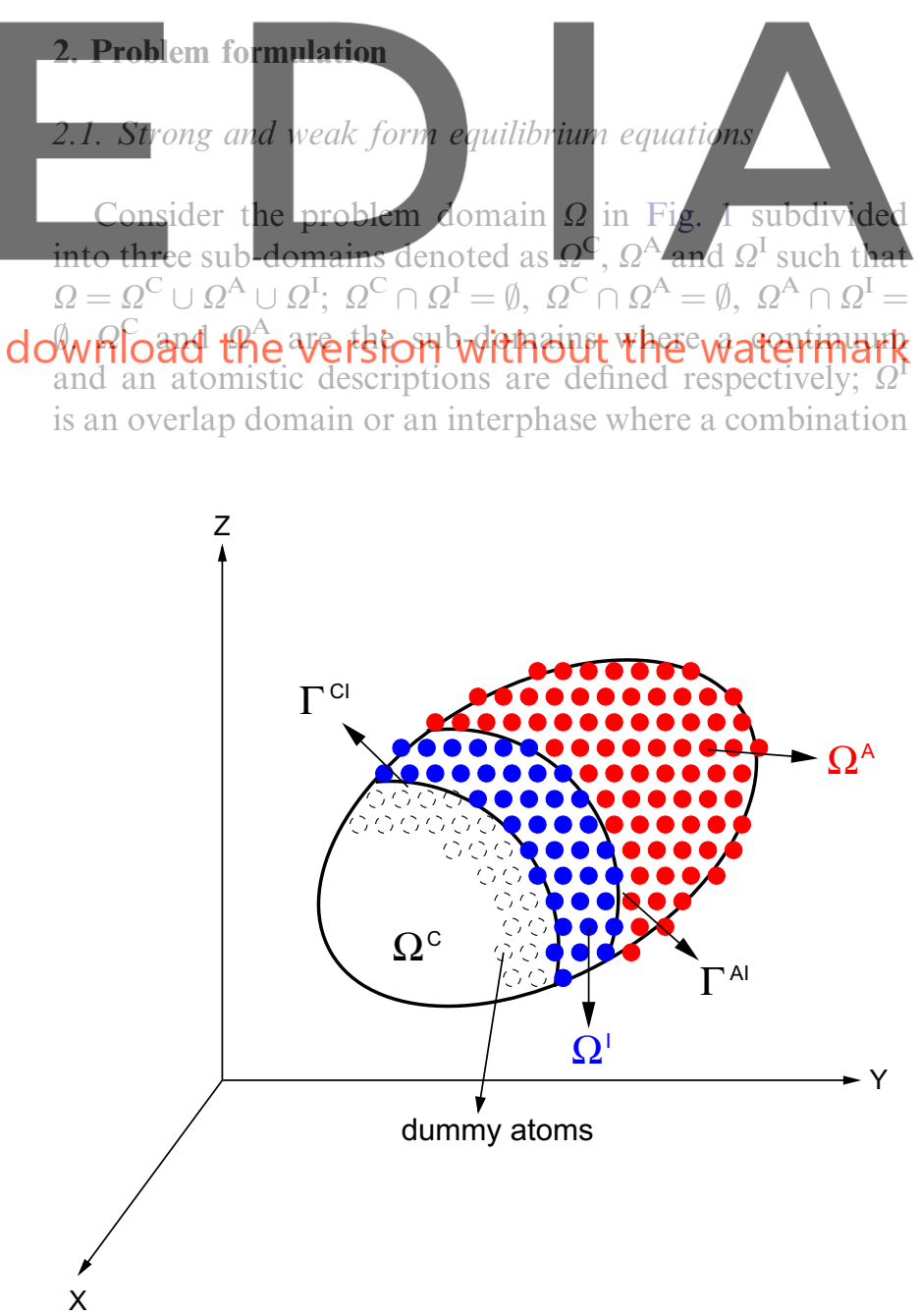

Fig. 1. Hybrid atomistic-continuum domain. 
of the atomistic and the continuum descriptions is defined. Dummy atoms shown in Fig. 1 are present in a region $r_{\mathrm{C}}$ distance away from $\Gamma^{\mathrm{CI}}$, where $r_{\mathrm{C}}$ is the cutoff distance of the inter-atomic potential. These dummy atoms are necessary to compute forces correctly on the atoms in $\Omega^{\mathrm{I}}$ close to $\Gamma^{\mathrm{CI}}$.

In the continuum sub-domain $\Omega^{\mathrm{C}}$ the governing equilibrium equation is

$\sigma_{i j, j}+b_{i}=0 \quad \mathbf{x} \in \Omega^{\mathrm{C}}$

along with appropriate boundary conditions, constitutive and kinematic equations. Lower case Roman subscripts $i, j, \ldots$ denote coordinates in the deformed configuration or current configuration, and comma in Eq. (1) denotes derivative with respect to space. Summation convention over the repeated indices is employed. $\sigma_{i j}$ and $b_{i}$ are the Cauchy stress tensor and the body force per unit volume respectively.

In the atomistic domain $\Omega^{\mathrm{A}}$ the equilibrium equation can be written as

$\left\{f_{i \alpha}+b_{i \alpha}\right\} \delta\left(\mathbf{x}-\mathbf{x}_{\alpha}\right)=0 \quad \mathbf{x} \in \Omega^{\mathrm{A}}$
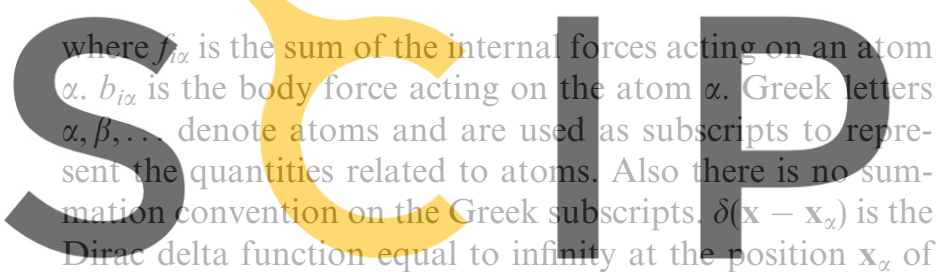
an atom $\alpha$ and zero elsewhere; its integral over the problem

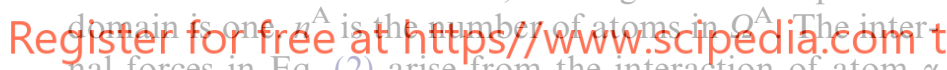
nal forces in Eq. (2) arise from the interaction of atom $\alpha$ with its neighbors depending on the inter-atomic potential used. An Embedded atom method (EAMI) potential is adopted here [10], although this is not a limitation of the method presented here. In EAM the total energy $\Phi$ of a system of atoms is obtained as the sum of energies of individual atoms $\Phi_{\alpha}$

$\Phi=\sum_{\alpha}^{n} \Phi_{\alpha}$

where $n$ is the total number of atoms in the system. $\Phi_{\alpha}$ is given by

$\Phi_{\alpha}=E\left(\rho_{\alpha}\right)+\frac{1}{2} \sum_{\beta, \beta \neq \alpha}^{\text {neig }^{\alpha}} V\left(r_{\alpha \beta}\right)$

$\rho_{\alpha}=\sum_{\beta, \beta \neq \alpha}^{\operatorname{neig}^{\alpha}} \Psi\left(r_{\alpha \beta}\right)$

where $\rho_{\alpha}$ is the total electron density at atom $\alpha, E\left(\rho_{\alpha}\right)$ is the embedding energy function. $r_{\alpha \beta}=\left|\mathbf{x}_{\alpha}-\mathbf{x}_{\beta}\right|$ is the distance between the atoms $\alpha$ and $\beta . V\left(r_{\alpha \beta}\right)$ is the pair potential term and $\Psi\left(r_{\alpha \beta}\right)$ is the electron density function, which have a cutoff distance in terms of $r$ as defined by the inter-atomic potential. Thus the summation in Eqs. (4) and (5) is over the atoms in a neighborhood of the atom $\alpha$ denoted by nei$\mathrm{g}^{\alpha}$. The internal force $f_{i \alpha}$ acting on an atom $\alpha$ in terms of the EAM potential is given by

$f_{i \alpha}=\sum_{\beta}^{n} \frac{\partial \Phi_{\beta}}{\partial d_{i \alpha}}$

where $d_{i \alpha}$ is the displacement of the atom $\alpha$ and

$\frac{\partial \Phi_{\beta}}{\partial d_{i \alpha}}=\frac{\partial E}{\partial \rho_{\beta}} \sum_{\gamma, \gamma \neq \beta}^{\text {neig }}\left\{\frac{\partial \Psi\left(r_{\beta \gamma}\right)}{\partial r_{\beta \gamma}} \frac{\partial r_{\beta \gamma}}{\partial d_{i \alpha}}\right\}+\frac{1}{2} \sum_{\gamma, \gamma \neq \beta}^{\text {neig }^{\beta}}\left\{\frac{\partial V\left(r_{\beta \gamma}\right)}{\partial r_{\beta \gamma}} \frac{\partial r_{\beta \gamma}}{\partial d_{i \alpha}}\right\}$

Note. $\Phi(r)$ and $V(r)$ are represented as a function of the distance $r$ and $E(\rho)$ as a function of the electron density $\rho$, both $r$ and $\rho$ being scalar parameters. The function values are interpolated by a 1-D cubic spline $\chi(x)$, where $\chi(x)$ denotes $\Psi(r)$ or $V(r)$ or $E(\rho)$ with $x$ being either $r$ or $\rho$. Cubic spline function is given by

$$
\begin{aligned}
\chi(x)= & y(i)+b(i)\{x-x(i)\}+c(i)\{x-x(i)\}^{2} \\
& +d(i)\{x-x(i)\}^{3}, \quad \forall x(i) \leqslant x<x(i+1)
\end{aligned}
$$

where $x(i)$ is the $i$ th spline knot position, $y(i)$ is the function value at the knot, $b(i), c(i)$ and $d(i)$ are the derivative coef-
fieients that construet the spline function. Spline parame-
ters are obtained through o leas square fit to both
experimental data and quantum mechanical force calcula-
tions. The optimized parameters that we have used are
avaitable at the website 1 ].
The motivation for using a blend wetween the continuum stress and the atomic force in the interphase $\Omega^{\mathrm{I}}$ comes

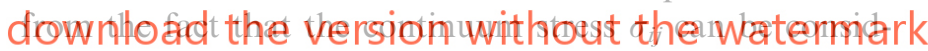
ered as an equivalent of the Virial stress $\tilde{\sigma}_{i j}$ defined at the atomistic level [35]. The Virial stress $\tilde{\sigma}_{i j}$ evaluated at an atom $\alpha$ is given in terms of the forces between its neighbors as follows [25]:

$\tilde{\sigma}_{i j}=\frac{1}{v} \sum_{\beta, \beta \neq \alpha}^{\mathrm{neig}^{\alpha}} r_{i \alpha \beta} f_{j \alpha \beta} ; \quad f_{j \alpha \beta}=\frac{\partial \Phi_{\beta}}{\partial d_{j \alpha}}$

where $r_{i \alpha \beta}=x_{i \alpha}-x_{i \beta}$ is the distance vector between the atoms $\alpha$ and $\beta, v$ is the volume of the unit cell associated with the atom $\alpha$. Blending the continuum stress $\sigma_{i j}$ is therefore equivalent to blending the atomistic force $f_{i \alpha \beta}$.

Thus in the interphase $\Omega^{\mathrm{I}}$, the equilibrium equation is obtained as follows:

$$
\begin{aligned}
& \left\{\Theta^{\mathrm{C}}(\mathbf{x}) \sigma_{i j}\right\}_{, j}+\Theta^{\mathrm{C}}(\mathbf{x}) b_{i} \\
& \quad+\sum_{\alpha}^{n^{\mathrm{I}}}\left[\left\{\sum_{\beta}^{\mathrm{neig}^{\alpha}}\left(\Theta_{\alpha \beta}^{\mathrm{A}} f_{i \alpha \beta}\right)+\Theta_{\alpha}^{\mathrm{A}} b_{i \alpha}\right\} \delta\left(\mathbf{x}-\mathbf{x}_{\alpha}\right)\right]=0 \quad \mathbf{x} \in \Omega^{\mathrm{I}}
\end{aligned}
$$

where

$$
\begin{aligned}
& \Theta_{\alpha}^{\mathrm{A}}=1-\Theta^{\mathrm{C}}\left(\mathbf{x}_{\alpha}\right) \\
& \Theta_{\alpha, \beta}^{\mathrm{A}}=1-\frac{1}{2}\left\{\Theta^{\mathrm{C}}\left(\mathbf{x}_{\alpha}\right)+\Theta^{\mathrm{C}}\left(\mathbf{x}_{\beta}\right)\right\}
\end{aligned}
$$


and $n^{\mathrm{I}}$ is the number of atoms in the interphase. The continuum blend function $\Theta^{\mathrm{C}}(\mathbf{x})$ is evaluated based on the proximity of the point $\mathbf{x} \in \Omega^{\mathrm{I}}$ to the boundaries $\Gamma^{\mathrm{CI}}$ and $\Gamma^{\mathrm{AI}}$ (Fig. 1). For instance $\Theta^{\mathrm{C}}(\mathbf{x})=1$ on $\Gamma^{\mathrm{CI}}$ and $\Theta^{\mathrm{C}}(\mathbf{x})=0$ on $\Gamma^{\mathrm{AI}}$. By defining $s \in[0,1]$ as a normalized distance in the physical domain from $\Gamma^{\mathrm{CI}}$ to $\Gamma^{\mathrm{AI}}, \Theta^{\mathrm{C}}(s)$ can be approximated to be a function of the scalar parameter $s$. To this end it is convenient to define the equilibrium Eq. (10) on the whole problem domain by defining $\Theta^{\mathrm{C}}(\mathbf{x})=0$ on $\Omega^{\mathrm{A}}$ and $\Theta^{\mathrm{C}}(\mathbf{x})=1$ on $\Omega^{\mathrm{C}}$.

There are several different possibilities for choosing $\Theta^{\mathrm{C}}$ over $\Omega^{\mathrm{I}}$. It can be approximated to coincide with 1-D linear shape function in $s$. Alternatively a $C^{1}$ continuous function can be constructed using a cubic polynomial satisfying the following conditions:

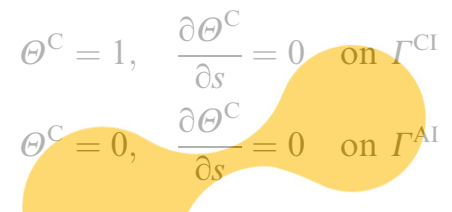

In addition to the equilibrium, compatibility needs to be satisfied between the atomistic and the continuum displacements in some average sense, schematically denoted as
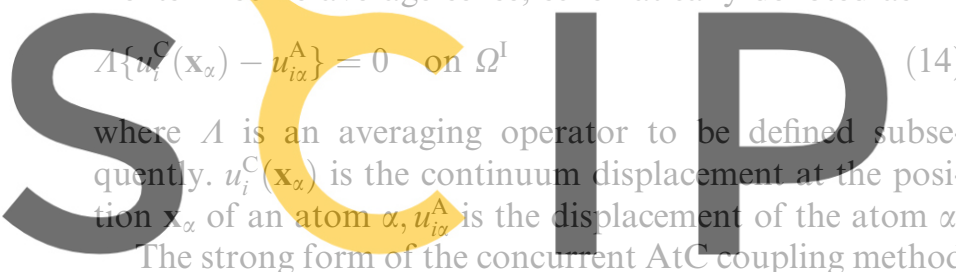

is formulated as: Given $b_{i}: \Omega^{\mathrm{C}} \cup \Omega^{\mathrm{I}} \rightarrow \mathfrak{R}, b_{i \alpha}: \Omega^{\mathrm{A}} \cup \Omega$

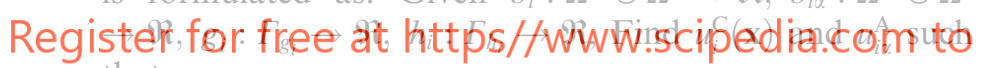

that

$$
\begin{aligned}
& \left\{\Theta^{\mathrm{C}}(\mathbf{x}) \sigma_{i j}\right\}_{, j}+\Theta^{\mathrm{C}}(\mathbf{x}) b_{i} \\
& \quad+\sum_{\alpha}^{n}\left[\left\{\sum_{\beta}^{\mathrm{neig} \mathrm{g}^{\alpha}}\left(\Theta_{\alpha \beta}^{\mathrm{A}} f_{i \alpha \beta}\right)+\Theta_{\alpha}^{\mathrm{A}} b_{i \alpha}\right\} \delta\left(\mathbf{x}-\mathbf{x}_{\alpha}\right)\right]=0 \quad \mathbf{x} \in \Omega
\end{aligned}
$$

$u_{i}^{\mathrm{C}}=g_{i} \quad$ on $\Gamma_{g_{i}}$

$\sigma_{i j} n_{j}=h_{i} \quad$ on $\Gamma_{h_{i}}$

$\Lambda\left\{u_{i}^{\mathrm{C}}\left(\mathbf{x}_{\alpha}\right)-u_{i \alpha}^{\mathrm{A}}\right\}=0 \quad \mathbf{x} \in \Omega^{\mathrm{I}}$

with appropriate constitutive equations and inter-atomic potentials. $n=n^{\mathrm{A}}+n^{\mathrm{I}}$ is the total number of atoms in the system. $g_{i}$ and $h_{i}$ are essential and natural boundary conditions on essential boundary $\Gamma_{g_{i}}$ and natural boundary $\Gamma_{h_{i}}$, respectively, where $\Gamma_{g_{i}} \cup \Gamma_{h_{i}}=\Gamma$ and $\Gamma_{g_{i}} \cap \Gamma_{h_{i}}=\emptyset$. Note that the equilibrium Eq. (15) is satisfied point-wise over $\Omega$, whereas the compatibility equation is defined in an average sense.

The weak form of the equilibrium Eq. (15) is stated as: Given $\quad b_{i}: \Omega^{\mathrm{C}} \cup \Omega^{\mathrm{I}} \rightarrow \mathfrak{R}, b_{i \alpha}: \Omega^{\mathrm{A}} \cup \Omega^{\mathrm{I}} \rightarrow \mathfrak{R}, g_{i}: \Gamma_{g_{i}} \rightarrow \mathfrak{R}$, $h_{i}: \Gamma_{h_{i}} \rightarrow \mathfrak{R}$, Find displacements $u_{i}^{\mathrm{C}}(\mathbf{x}) \in \mathscr{U}_{i}^{\mathrm{C}} \quad$ and $u_{i \alpha}^{\mathrm{A}} \in \mathscr{U}_{i}^{\mathrm{A}}$ such that

$$
\begin{aligned}
& \int_{\Omega} w_{i}^{\mathrm{C}}\left\{\left(\Theta^{\mathrm{C}} \sigma_{i j}\right)_{j}+\Theta^{\mathrm{C}} b_{i}\right\} \mathrm{d} \Omega \\
& \quad+\int_{\Omega} w_{i \alpha}^{\mathrm{A}}\left\{\sum_{\alpha}^{n}\left[\sum_{\beta}^{\mathrm{neig} \alpha}\left(\Theta_{\alpha \beta}^{\mathrm{A}} f_{i \alpha \beta}\right)+\Theta_{\alpha}^{\mathrm{A}} b_{i \alpha} \delta\left(\mathbf{x}-\mathbf{x}_{\alpha}\right)\right]\right\} \mathrm{d} \Omega=0 \\
& \forall w_{i}^{\mathrm{C}} \in \mathscr{W}_{i}^{\mathrm{C}}, \quad \forall w_{i \alpha}^{\mathrm{A}} \in \mathscr{W}_{i}^{\mathrm{A}}
\end{aligned}
$$

Integration by parts of the first term in Eq. (19) results in

$$
\begin{aligned}
& -\int_{\Omega} w_{i, j}^{\mathrm{C}} \Theta^{\mathrm{C}} \sigma_{i j} \mathrm{~d} \Omega+\int_{\Gamma_{h_{i}}} w_{i}^{\mathrm{C}} \Theta^{\mathrm{C}} h_{i} \mathrm{~d} \Gamma+\int_{\Omega} w_{i}^{\mathrm{C}} \Theta^{\mathrm{C}} b_{i} \mathrm{~d} \Omega \\
& +\int_{\Omega} \sum_{\alpha}^{n} w_{i \alpha}^{\mathrm{A}}\left[\sum_{\beta}^{\mathrm{neig}^{\alpha}}\left(\Theta_{\alpha \beta}^{\mathrm{A}} f_{i \alpha \beta}\right)+\Theta_{\alpha}^{\mathrm{A}} b_{i \alpha}\right] \delta\left(\mathbf{x}-\mathbf{x}_{\alpha}\right) \mathrm{d} \Omega=0
\end{aligned}
$$

\section{The weak compatibility $\Lambda$ is defined as follows:}
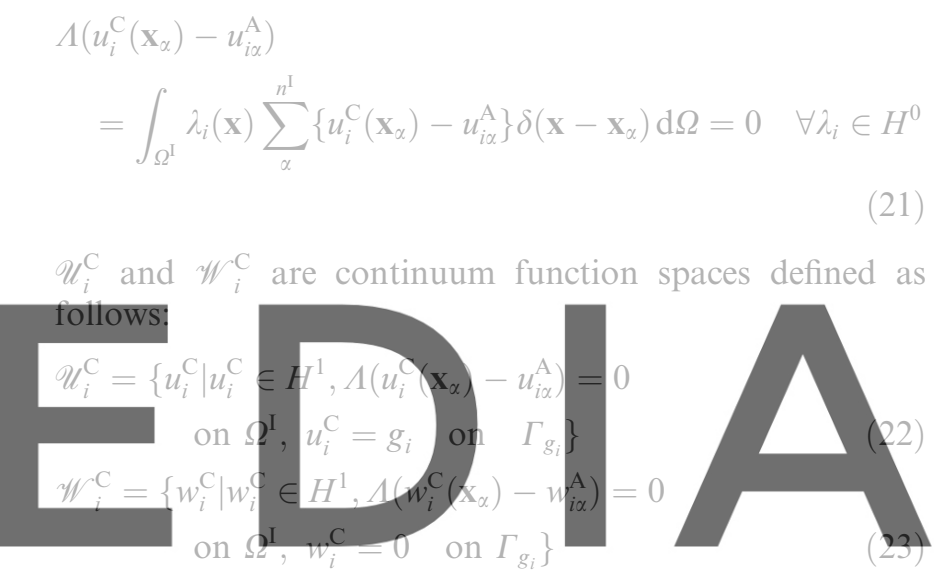

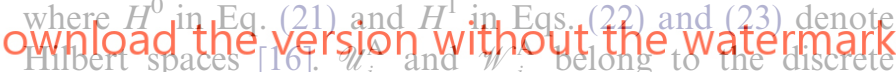

phase space of the atomistic system given by

$$
\begin{aligned}
& \mathscr{U}_{i}^{\mathrm{A}}=\left\{u_{i \alpha}^{\mathrm{A}} \mid u_{i \alpha}^{\mathrm{A}} \in \mathfrak{R}^{n}, \Lambda\left(u_{i}^{\mathrm{C}}\left(\mathbf{x}_{\alpha}\right)-u_{i \alpha}^{\mathrm{A}}\right)=0 \quad \text { on } \Omega^{\mathrm{I}}\right\} \\
& \mathscr{W}_{i}^{\mathrm{A}}=\left\{w_{i \alpha}^{\mathrm{A}} \mid w_{i \alpha}^{\mathrm{A}} \in \mathfrak{R}^{n}, \Lambda\left(w_{i}^{\mathrm{C}}\left(\mathbf{x}_{\alpha}\right)-w_{i \alpha}^{\mathrm{A}}\right)=0 \quad \text { on } \Omega^{\mathrm{I}}\right\}
\end{aligned}
$$

Weight functions $w_{i}^{\mathrm{C}}$ and $w_{i \alpha}^{\mathrm{A}}$ are related through the compatibility condition.

\subsection{Discretized equations of equilibrium and constraints}

A finite element discretization of the problem domain $\Omega$ is denoted by $\Omega^{h}$. The continuum displacement and test functions defined over $\Omega^{\mathrm{C}} \cup \Omega^{\mathrm{I}}$ are discretized using $C^{0}$ continuous finite element shape functions. The discretized displacement is denoted by $u_{i}^{h} \in \mathscr{U}_{i}^{h}$ and the discretized test function is denoted by $w_{i}^{h} \in \mathscr{W}_{i}^{h}$. The spaces $\mathscr{U}_{i}^{h}$ and $\mathscr{W}_{i}^{h}$ are given by

$$
\begin{aligned}
\mathscr{U}_{i}^{h}=\left\{u_{i}^{h} \mid u_{i}^{h}=N_{B} d_{i B}^{\mathrm{C}}, \Lambda^{h}\left(u_{i}^{h}\left(\mathbf{x}_{\alpha}\right)-u_{i \alpha}^{\mathrm{A}}\right)=0\right. \\
\\
\text { on } \left.\Omega^{h^{\mathrm{I}}}, u_{i}^{h}=g_{i} \text { on } \Gamma_{g_{i}}^{h}\right\} \\
\mathscr{W}_{i}^{h}=\left\{w_{i}^{h} \mid w_{i}^{h}=N_{B} a_{i B}^{\mathrm{C}}, \Lambda^{h}\left(w_{i}^{h}\left(\mathbf{x}_{\alpha}\right)-w_{i \alpha}^{\mathrm{A}}\right)=0\right. \\
\text { on } \left.\Omega^{h^{\mathrm{I}}}, w_{i}^{h}=0 \text { on } \Gamma_{g_{i}}^{h}\right\}
\end{aligned}
$$


where $N_{B}$ are the finite element shape functions associated with the finite element nodes $B, d_{i B}^{\mathrm{C}}$ are the nodal degrees of freedom and $a_{i B}^{\mathrm{C}}$ are the nodal multipliers corresponding to test functions. Summation convention over repeated index $B$ is employed.

The discrete compatibility equation is constructed by discretizing $\lambda(\mathbf{x}) \in H^{0}$ using piecewise constant shape functions defined to be constant over the finite element domains $\Omega^{e} \in \Omega^{h^{1}}$

$\lambda_{i}^{h}(\mathbf{x})=\sum_{\Omega^{e} \in \Omega^{h^{1}}} N^{e} \zeta_{i}^{e}$

where

$N^{e}= \begin{cases}1 & \text { on } \Omega^{e} \\ 0 & \text { elsewhere }\end{cases}$

Substituting Eqs. (28) and (29) into Eq. (21) we obtain

$\sum_{\Omega^{e} \in \Omega^{h^{1}}} \int_{\Omega^{e}} \zeta_{i}^{e} \sum_{\alpha}^{n^{\mathrm{I}}}\left\{u_{i}^{\mathrm{C}}\left(\mathbf{x}_{\alpha}\right)-u_{i \alpha}^{\mathrm{A}}\right\} \delta\left(\mathbf{x}-\mathbf{x}_{\alpha}\right) \mathrm{d} \Omega=0$

Requiring the arbitrariness of $\zeta_{i}^{e}$ yields the following discrete compatibility equation for every element $\Omega^{e}, n^{e}$ being the number of atoms in an element $\Omega^{e}$ :
$\Lambda^{\prime \prime}\left\{u_{i}^{h}\left(\mathbf{x}_{\alpha}\right)-u_{i \alpha}^{\mathrm{A}}\right\}=\sum_{\alpha}^{n^{e}}\left\{N_{B}\left(\mathbf{x}_{\alpha}\right) d_{i B}^{\mathrm{C}}-u_{i \alpha}^{\mathrm{A}}\right\}=0 \quad \forall \Omega^{e} \quad(\beta 1)$
The above Eq. (31) yields number of constraint equations
equal to the number of spatial dimension for each finite element $\Omega^{e} \in \Omega^{h^{1}}$. From Eq. (31) the degrees of freedom of

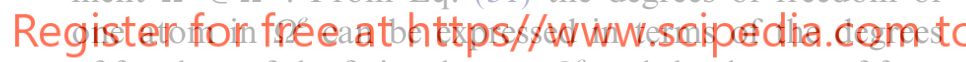
of freedom of the finite element $\Omega^{e}$ and the degrees of freedom of the remaining atoms in the element. Note that at least one atom has to be positioned with an element $\Omega^{e}$ in the interphase.

Discretized continuum weight functions $w_{i}^{h}$ are related to atomistic weight functions through discretized compatibility Eq. (30). By constructing the compatibility equations such that the atoms within an element $\Omega^{e} \in \Omega^{h^{1}}$ are enslaved by the continuum degrees of freedom of that element (as in the case of the local quasicontinuum method), discretized continuum weight functions $w_{i}^{h}$ computed at the position of atoms coincide with the atomistic weight functions $w_{i \alpha}^{\mathrm{A}}$.

Let $d_{i P}$ be the independent degrees of freedom in a concurrent problem formulation. ${ }^{2}$ Finite element degrees of freedom over $\Omega^{h^{\mathrm{C}}} \cup \Omega^{h^{1}}$ are denoted by $d_{j D}^{\mathrm{C}}$. Master (independent) atomistic degrees of freedom over $\Omega^{\mathrm{A}} \cup \Omega^{h^{1}}$ are denoted by $d_{j \alpha}^{\mathrm{A}}$. Let $T_{j D i P}^{\mathrm{C}}$ and $T_{j \alpha i P}^{\mathrm{A}}$ be the transformation matrices consisting of zeros and ones such that

$d_{j D}^{\mathrm{C}}=T_{j D i P}^{\mathrm{C}} d_{i P} ; \quad d_{j \alpha}^{\mathrm{A}}=T_{j \alpha i P}^{\mathrm{A}} d_{i P}$

\footnotetext{
${ }^{2}$ Subscripts $P, Q, R$, etc. are used to denote global degrees of freedom (combined atomistic and continuum), where as subscripts $A, B, C, D$, etc. are used to denote the finite element nodal degrees of freedom.
}

$T_{j D i P}^{\mathrm{C}}$ and $T_{j \alpha i P}^{\mathrm{A}}$ are used for writing convenience that allow the system of equations to be written in terms of total independent degrees of freedom for the whole problem $d_{i P}$ rather than writing partitioned system of equations in terms of partitioned degrees of freedom $d_{j D}^{\mathrm{C}}$ and $d_{j \alpha}^{\mathrm{A}}$. Similarly the multipliers of the test function are related as

$a_{j D}^{\mathrm{C}}=T_{j D i P}^{\mathrm{C}} a_{i P} ; a_{j \alpha}^{\mathrm{A}}=T_{j \alpha i P}^{\mathrm{A}} a_{i P}$

where $a_{i P}$ are the global independent multipliers of the test function, $a_{j D}^{\mathrm{C}}$ and $a_{j \alpha}^{\mathrm{A}}$ are the multipliers of the test function corresponding to the degrees of freedom $d_{j D}^{\mathrm{C}}$ and $d_{j \alpha}^{\mathrm{A}}$.

The discretized system of equations shown below is obtained by using Eqs. (26)-(33) in the weak form equilibrium Eq. (20)
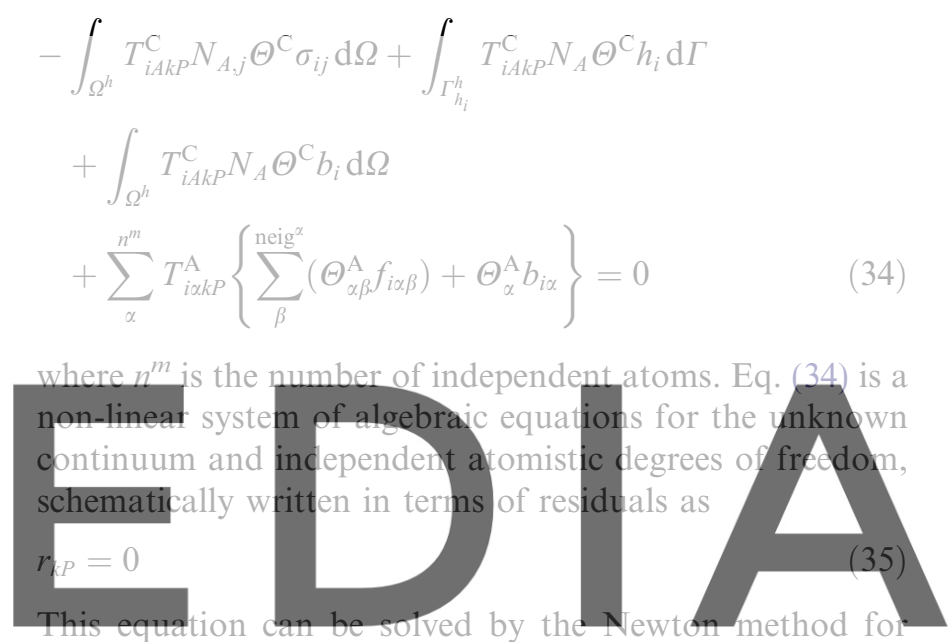

each load increment. The tangent stiffness matrix is given

döwnload the version without the watermark

$$
\mathscr{K}_{k P M Q}=\frac{\partial r_{k P}}{\partial d_{m Q}} \equiv \frac{\partial \dot{r}_{k P}}{\partial \dot{d}_{m Q}}
$$

Superimposed dot in Eq. (36) represents the material time derivative. Assuming no follower forces and no body forces simplifies Eq. (34) to

$$
\begin{aligned}
r_{k P}= & -\int_{\Omega^{h}} T_{i A k P}^{\mathrm{C}} N_{A, j} \Theta^{\mathrm{C}} \sigma_{i j} \mathrm{~d} \Omega \\
& +\sum_{\alpha}^{n^{m}} T_{i \alpha k P}^{\mathrm{A}}\left\{\sum_{\beta}^{\mathrm{neig}^{\alpha}}\left(\Theta_{\alpha \beta}^{\mathrm{A}} f_{i \alpha \beta}\right)\right\}=0
\end{aligned}
$$

Consistent linearization of the first term of the tangent stiffness matrix in Eq. (37) yields

$$
\begin{aligned}
\dot{r}_{k P}^{(1)} & =\frac{\mathrm{d}}{\mathrm{d} t}\left\{\int_{\Omega^{h}} T_{i A k P}^{\mathrm{C}} \frac{\partial N_{A}}{\partial x_{j}} \Theta^{\mathrm{C}} \sigma_{i j} \mathrm{~d} \Omega\right\} \\
& =\int_{\Omega_{0}^{h}} T_{i A k P}^{\mathrm{C}} \frac{\partial N_{A}}{\partial X_{\mathrm{I}}} \Theta^{\mathrm{C}} \frac{\mathrm{d}}{\mathrm{d} t}\left\{\frac{\partial X_{\mathrm{I}}}{\partial x_{j}} \sigma_{i j} J\right\} \mathrm{d} \Omega_{0}
\end{aligned}
$$

$\Omega_{0}$ denotes the initial or undeformed configuration, $X_{\mathrm{I}}$ is the initial position with big Roman subscripts denoting the coordinates in the initial configuration. $\Theta^{\mathrm{C}}$ is expressed as a function of $X_{\mathrm{I}} \cdot \frac{\partial X_{I}}{\partial x_{j}}$ is the inverse of the deformation gradient tensor. $J$ is the determinant of the Jacobian such 
that $\mathrm{d} \Omega=J \mathrm{~d} \Omega_{0}$. Simplification of the derivative terms in Eq. (38) gives

$\dot{r}_{k P}^{(1)}=\int_{\Omega^{h}} T_{i A k P}^{\mathrm{C}} N_{A, j} \Theta^{\mathrm{C}}\left\{\dot{\sigma}_{i j}+\left(\sigma_{i j} \delta_{k l}-\sigma_{i l} \delta_{j k}\right) \dot{u}_{k, l}^{\mathrm{C}}\right\} \mathrm{d} \Omega$

Linearization of the Cauchy stress depends on the choice of material model and the material and rotational stress update. Details can be found in Refs. [4,36]. The term in parenthesis in Eq. (39) can be expressed as

$$
\begin{aligned}
\dot{\sigma}_{i j}+\left(\sigma_{i j} \delta_{k l}-\sigma_{i l} \delta_{j k}\right) \dot{u}_{k, l}^{\mathrm{C}} & =\mathscr{L}_{i j k l} \dot{u}_{k, l}^{\mathrm{C}}=\mathscr{L}_{i j k l} N_{B, l} \dot{d}_{k B}^{\mathrm{C}} \\
& =\mathscr{L}_{i j k l} N_{B, l} T_{k B n R}^{\mathrm{C}} \dot{d}_{n R}
\end{aligned}
$$

where $\mathscr{L}_{i j k l}$ depends on the material model and algorithmic parameters. By substituting Eq. (40) into Eq. (39) it can be seen that the resulting tangent stiffness matrix is symmetric if $\mathscr{L}_{i j k l}$ has a major symmetry. Consistent linearization of the second term of the tangent stiffness matrix in Eq. (37) yields

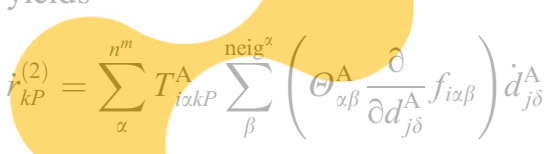
$=\left(\sum^{n^{m}}+T^{\wedge} \sum^{\text {neig }}\left(\begin{array}{ll}\wedge & \partial\end{array}\right)\right.$

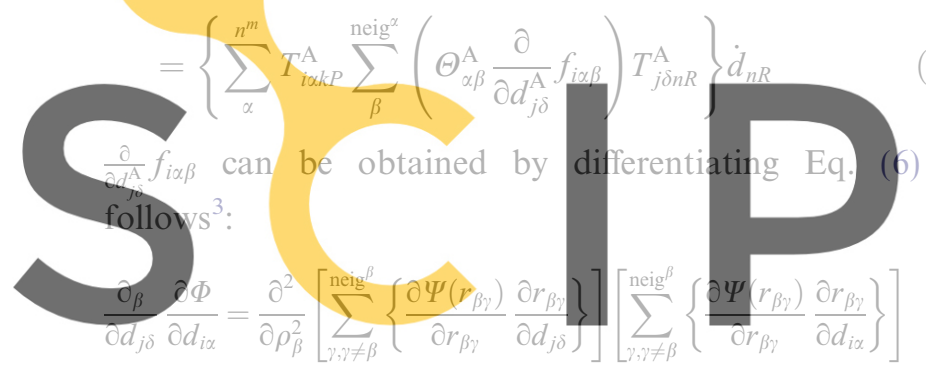

$(41)$

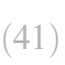

problem discussed in Section 2.4 and in [3]. However handling of ghost forces discussed with Fig. 1 will be difficult for a piecewise constant blend.

2. Piecewise linear blend function: Local coordinates of the parent domain of tetrahedral mesh elements is used to construct linear blend function. With $u=1-r-s-t$ linear blend function is of the form

$$
\Theta^{\mathrm{C}}(r, s, t, u)=A_{1} r+A_{2} s+A_{3} t
$$

Constants $A_{1}, A_{2}$ and $A_{3}$ are determined based on the normalized distance of the vertices from $\Gamma^{\mathrm{CI}}$ shown in Fig. 1. Piecewise linear blend is a common choice.

3. Piecewise cubic blend function: A cubic blend function is defined in the local coordinates of the parent domain of tetrahedral mesh elements. It is constructed from cubic Bézier patch for a tetrahedron analogous to the Bézier triangle [14]. A cubic Bézier tetrahedron is defined as

$$
\begin{gathered}
\Theta^{\mathrm{C}}(r, s, t, u)=\sum_{|a|=3}\left\{\frac{n !}{a ! b ! c ! d !} r^{a} s^{b} t^{c} u^{d} B_{(a b c d)}\right\} \\
|a|=a+b+c+d=3 \text { for cubic }
\end{gathered}
$$

where $a, b, c$ and $d$ are the indices of the control points

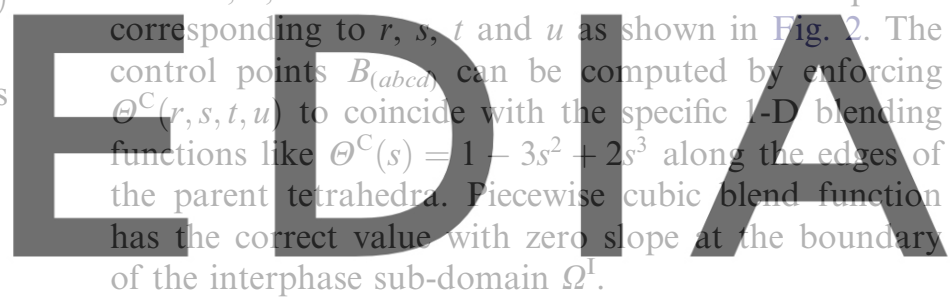

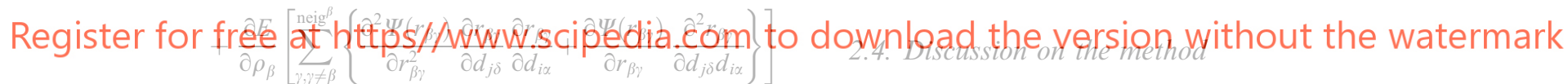

$+\frac{1}{2}\left[\sum_{\gamma, \gamma \neq \beta} \operatorname{neig}^{\beta}\left\{\frac{\partial^{2} V\left(r_{\beta \gamma}\right)}{\partial r_{\beta \gamma}^{2}} \frac{\partial r_{\beta \gamma}}{\partial d_{j \delta}} \frac{\partial r_{\beta \gamma}}{\partial d_{i \alpha}}+\frac{\partial V\left(r_{\beta \gamma}\right)}{\partial r_{\beta \gamma}} \frac{\partial^{2} r_{\beta \gamma}}{\partial d_{j \delta} d_{i \alpha}}\right\}\right]$

\subsection{Blend functions over a discretized domain}

The continuum sub-domain $\Omega^{\mathrm{C}}$ and the interphase subdomain $\Omega^{\mathrm{I}}$ are discretized into a finite element mesh with tetrahedral elements in 3D. We consider three different scenarios of the blend functions:

1. Piecewise constant blend function: In this scenario, a constant value for $\Theta^{\mathrm{C}}$ is assigned for each $\Omega^{e} \in \Omega^{h^{1}}$ based on the normalized distance of the centroid of $\Omega^{e}$ from $\Gamma^{\mathrm{CI}}$ in Fig. 1. $\Theta^{A}=1-\Theta^{\mathrm{C}}$ is a constant for the atoms bounded by an element $\Omega^{e} \in \Omega^{h^{1}}$. Piecewise constant blend is the simplest and does not have consistency

\footnotetext{
${ }^{3}$ The subscript $\delta$ used to denote an atom is not to be confused with the Dirac delta function.
}

A comparison of AtC with the similar existing methods is discussed in this section. An overlapping domain between the continuum and atomistic sub-domains has been used in the past to couple continuum and atomistic models. In the paper by Curtin and Miller [8] a review of

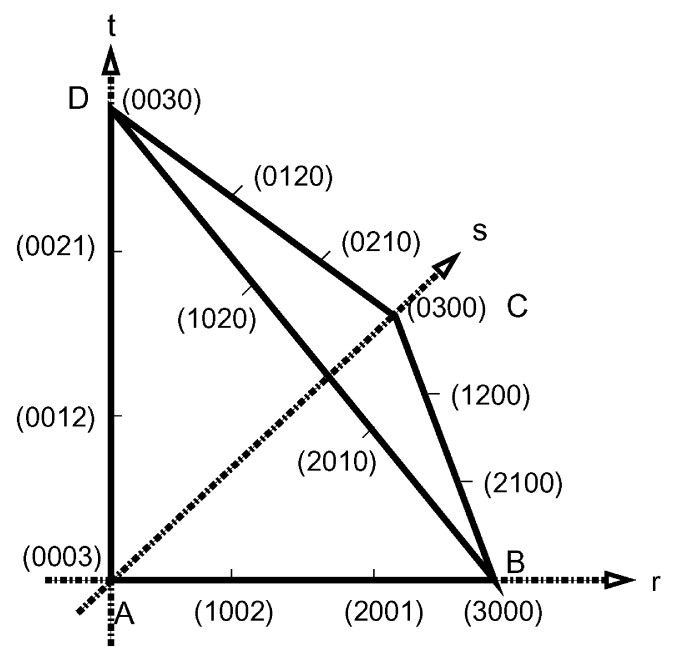

Fig. 2. Parent tetrahedron with indices of control points. 
different continuum-atomistic concurrent methods is given with an emphasis on the generalization of atomistic/continuum transition region. Details of the transition region are discussed for the quasicontinuum method [32,22], the coupling of length scales (CLS) method [6,2], the finite element atomistics (FEAt) method [19] and coupled atomistics and discrete dislocation (CADD) method [29] under a unified generic transition model as shown in Fig. 2 of [8]. It is evident from the discussion of transition region in [8] that all the methods discussed in there have a continuum mesh refined to the level of atomic spacing and the continuum nodes coincide with the atoms in the transition region. This is not necessary for the AtC method presented here as shown in Figs. 4, 6 and 8. In fact this may be a tedious extra work in an adaptive framework where the atomistic domain is not known a priori. The bridging domain method $[5,34]$ and the multiscale modeling method of Luan et al. [20] also eliminated the restriction of continuum mesh refinement down to atomistic spacing and coincidence of atoms with continuum nodes in the transition region.

Among the methods discussed in [8] some of the methods like the quasicontinuum method [32,22], CLS method $[6,2]$ have a well defined energy functional that approxi-

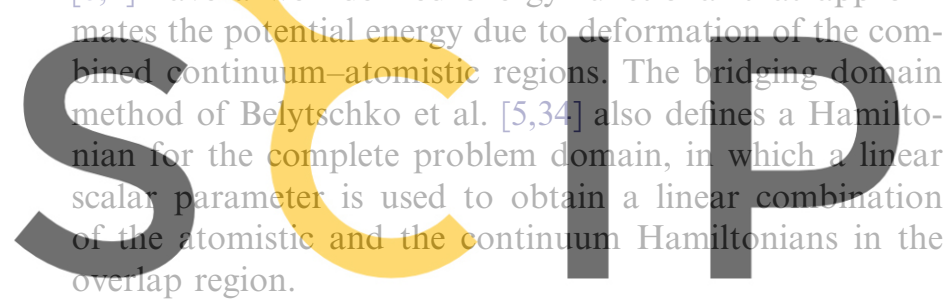

In the FEAt method [19] the solution is obtained over

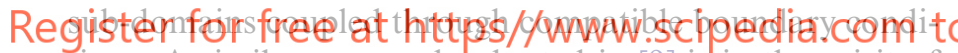
tions. A similar approach adopted in [9] is in the spirit of Schwarz overlapping method, which has two conditions for uniqueness and convergence (see for instance [7]). These are (i) convexity and (ii) use of identical mathematical model in the overlapping regions. In the absence of (i) there is no guarantee that the residuals vanish as the compatibility is enforced.

The method presented here minimizes residuals of Eq. (35) to bring unbalance forces to zero during the solution procedure. The solution is obtained in one step for the entire domain without using the interphase to transfer information from the continuum to the atomistic domain and vice versa as part of the iterative solution (as in [9]). The present method may also be used in situations in which devising an energy functional for the entire system may not be possible, for example in the presence of irreversible processes. Use of blending functions $\Theta^{\mathrm{C}}(\mathbf{x})$ and $\Theta^{\mathrm{A}}(\mathbf{x})$ leads to an approximation of the physically meaningful energy in the overlap interphase sub-domain.

The atomistic blend function $\Theta^{\mathrm{A}}$ is to be handled carefully due the non-local nature of the atomistics. Analyses of the AtC methods in [3] discusses the inconsistencies that can arise if $\Theta^{\mathrm{A}}$ is not treated carefully. To fix ideas consider a 1-D case of two interacting atoms in $\Omega^{\mathrm{I}}$ depicted in Fig. 3. If the blend functions in $\Omega^{\mathrm{I}}$ are defined in the traditional

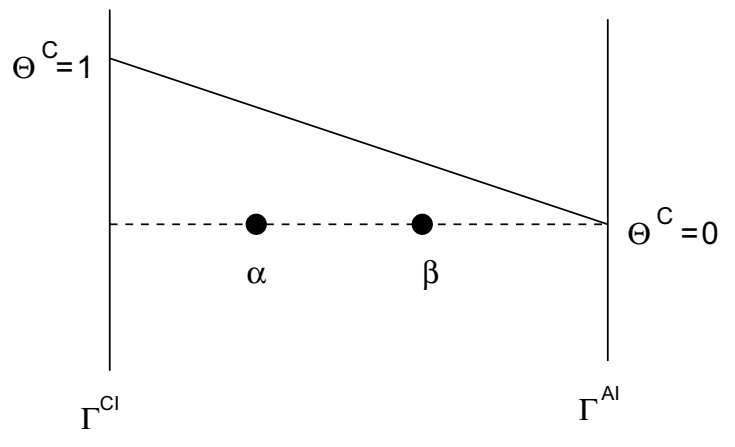

Fig. 3. Consistency check for AtC.

way as $\Theta^{\mathrm{A}}(\mathbf{x})=1-\Theta^{\mathrm{C}}(\mathbf{x})$ either the energy blend or the force blend leads to the following inconsistency. For instance, force at the position of the atom $\alpha$ is $\left\{1-\Theta^{\mathrm{C}}\left(\mathbf{x}_{\alpha}\right)\right\} f_{i \alpha \beta}$ and that at the position of the atom $\beta$ is $\left\{1-\Theta^{\mathrm{C}}\left(\mathbf{x}_{\beta}\right)\right\} f_{i \alpha \beta}$. Since $\Theta^{\mathrm{C}}\left(\mathbf{x}_{\alpha}\right) \neq \Theta^{\mathrm{C}}\left(\mathbf{x}_{\beta}\right)$ in general, $\left\{1-\Theta^{\mathrm{C}}\left(\mathbf{x}_{\alpha}\right)\right\} f_{i \alpha . \beta} \neq\left\{1-\Theta^{\mathrm{C}}\left(\mathbf{x}_{\beta}\right)\right\} f_{i \alpha \beta}$, which violates the Newton's third law and causes non-symmetric stiffness matrix. Incidentally a constant blend of $\Theta^{\mathrm{C}}=\Theta^{\mathrm{A}}=0.5$ used by Broughton et al. [6,2] is consistent in this sense,

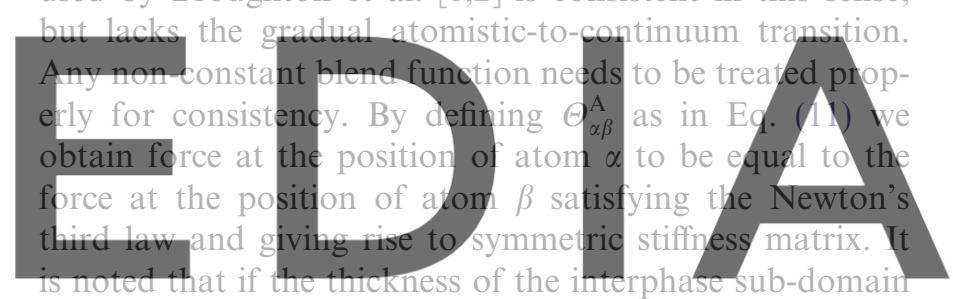

is reduced to zero, one recovers the residual definitions of

downdorat the peitsien 3 Without the watermark

In addition to equilibrium (Eqs. (15)-(17)), a compatibility is enforced (Eq. (18)) between the continuum solution and the atomistic solution. This is similar to the constraints imposed by the Lagrange multiplier or augmented Lagrangian method in $[5,34]$. However the compatibility in Eq. (18) allows for a flexibility in imposing the constraints between the continuum solution and atomistic solution. By controlling the discretization of $\lambda_{i}(\mathbf{x})$ in Eq. (28) we can control the strength of the coupling, which will be studied in detail in a continuing work.

\section{Patch tests}

A series of patch tests are conducted to verify the problem formulation and its implementation. Fig. 4 shows the hybrid atomistic-continuum domain considered for the patch tests. A cube with an atomistic sub-domain $\Omega^{\mathrm{A}}$ at the center and surrounded by an interphase sub-domain $\Omega^{\mathrm{I}}$ is subjected to 6 constant strain modes ( 3 normal strain modes and 3 shear strain modes) and 6 rigid body modes ( 3 translation and 3 rotation) one at a time. The problem domain $\Omega$ is discretized with a tetrahedral finite element mesh. The finite element nodes only in $\Omega^{h^{\mathrm{C}}} \cup \Omega^{h^{1}}$ contribute to the continuum residual equations. The EAM potential 


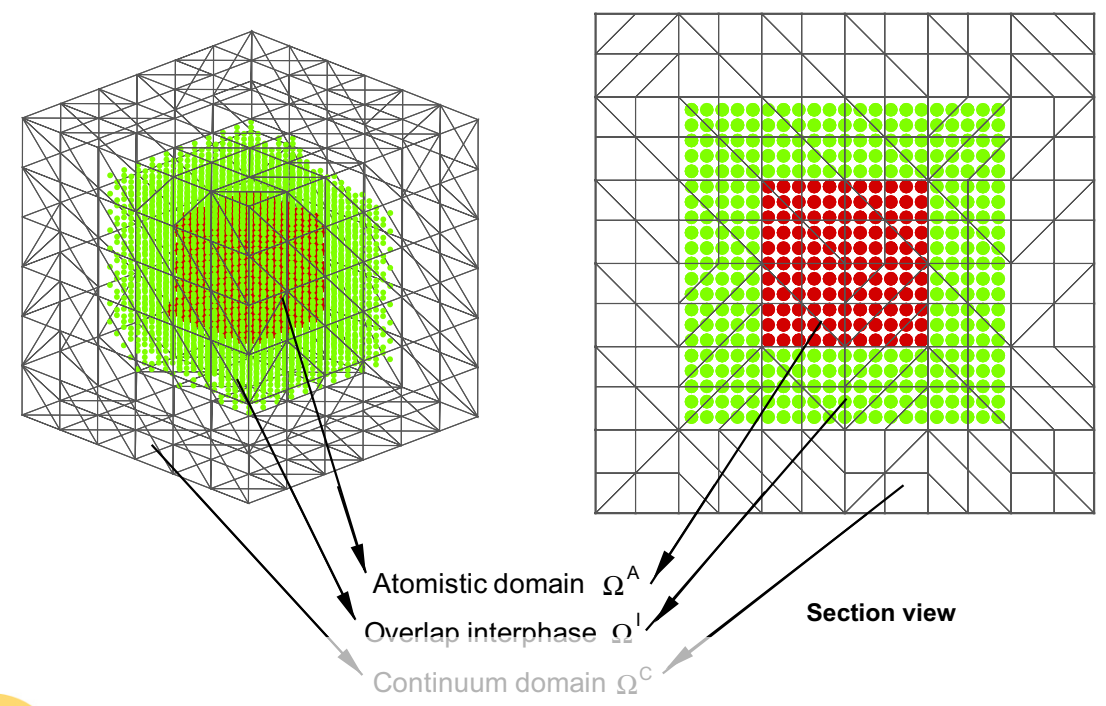

Fig. 4. Hybrid atomistic-continuum domain for patch tests.

for Aluminum [13] is chosen for the atomistics and a linear elastic constitutive relationship consistent with the EAM is chosen for the continuum [9]. The constant strain modes and the rigid body modes are imposed through the appro-
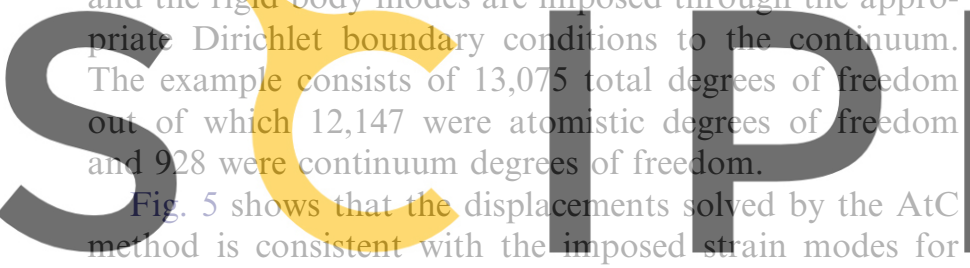

the case of a normal strain mode $\varepsilon_{x x}=0.001$ and a shear

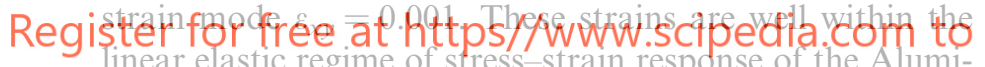
num lattice. The displacements were consistent for the other constant strain modes and rigid body modes as well.
Energy density is calculated for each element in the continuum sub-domain $\Omega^{h^{\mathrm{C}}}$, for each atom in the atomistic sub-domain $\Omega^{\mathrm{A}}$ and at each atom location in the overlap interphase $\Omega^{h^{1}}$. It was found to be accurate within a toler-

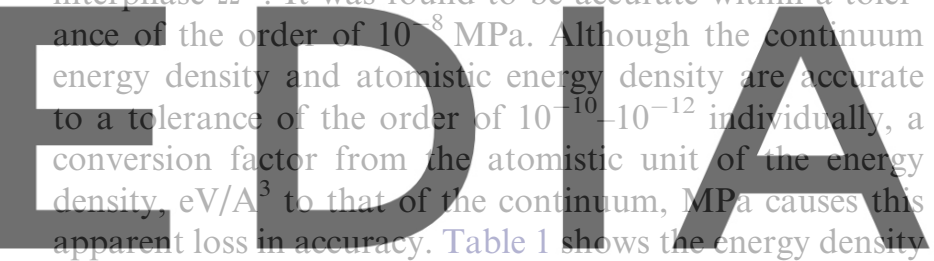
for different strain modes. The energy density for the rigid

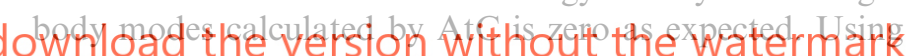
different blend functions discussed in Section 2.3 in the overlap interphase $\Omega^{\mathrm{I}}$ of the patch test domain (Fig. 4) did not affect the patch test resuits. (a) Displacement plot for $\varepsilon_{x x}=0.001$

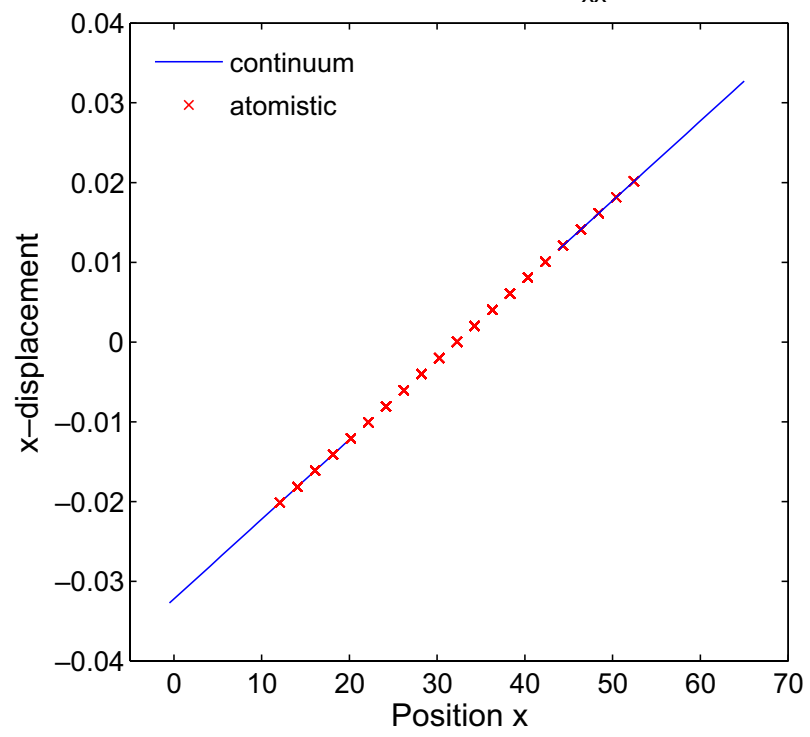

(b) Displacement plot for $\varepsilon_{x y}=0.001$

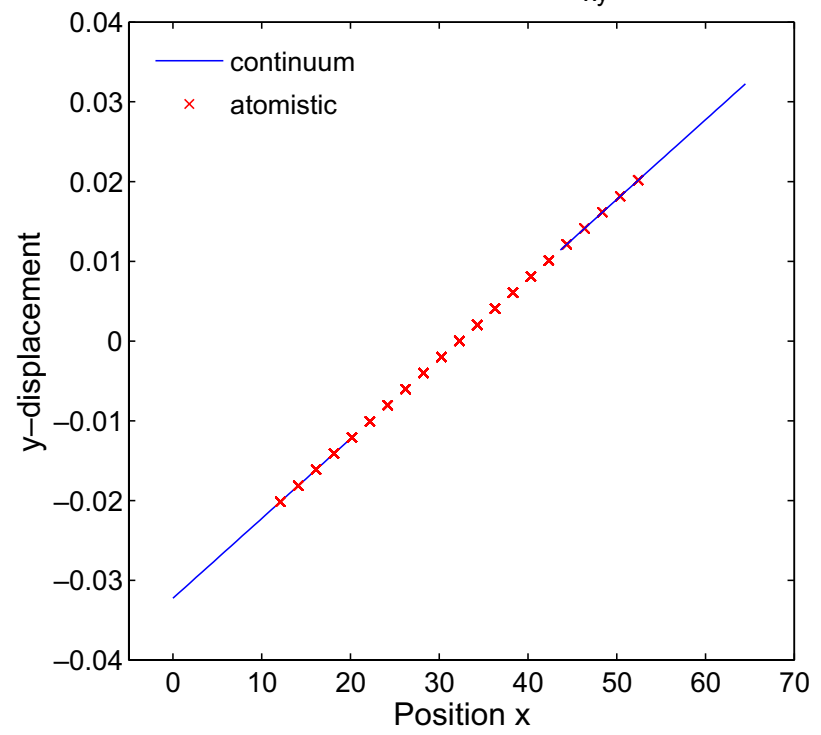

Fig. 5. Displacement along $X$ direction. 
Table 1

Strain energy density by AtC formulation

Strain modes

$\varepsilon_{x x}=0.001$

$\varepsilon_{y y}=0.001$

$\varepsilon_{z z}=0.001$

$\varepsilon_{x y}=0.001$

$\varepsilon_{y z}=0.001$

$\varepsilon_{y z}=0.001$

\section{Numerical examples}

Two numerical examples are illustrated in this section. The first one is nano-indentation of a thin film and the second is a nano-void subjected to hydrostatic loading. The continuum is linear elastic. The EAM potential of the Aluminum given by Ercolessi and Adams [13] is used for the atomistics and the elastic constitutive parameters chosen for the continuum are consistent with the EAM potential. A finite element discretization of the problem domain is constructed such that the mesh is finer in the regions where stresses are expected to be high. A hybrid atomistic-continuum concurrent model is constructed a priori by replacing the mesh elements in the regi
expected to be high with an ato
adaptive scheme by which the
selected based on the underlying
in a subsequent publication. In
paper there is also a discussion on
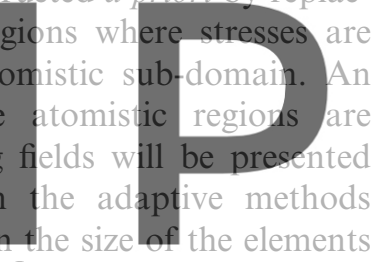

in the interphase sub-domain $\Omega^{\mathrm{I}}$. Although the entire

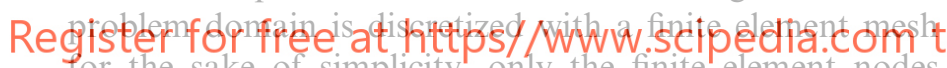
in the sub-domain $\Omega^{\mathrm{C}} \cup \Omega^{\mathrm{I}}$ contribute to the continuum residual in Eq. (34). Eq. (35) was solved by a non-linear multi-variable minimization library based on conjugate gradient algorithm.
Crystal defects such as dislocations that are formed as a result of loading are captured by the atomistic model. Centrosymmetry calculation [18] is used to detect the atoms in the dislocation core and these atoms are plotted to show the dislocation core structure. The centrosymmetry parameter for each atom is defined as follows:

$P=\sum_{\alpha=1}^{6}\left|\mathbf{R}_{\alpha}+\mathbf{R}_{\alpha+6}\right|^{2}$

where $\mathbf{R}_{\alpha}$ and $\mathbf{R}_{\alpha+6}$ are the vectors corresponding to the six pairs of opposite nearest neighbors in the FCC lattice. The centrosymmetry parameter is zero for the atoms in a perfect crystal. For $P=0.5-4.0$ the atom is considered to be located at a dislocation core [18]. Centrosymmetry criteria has been used to show dislocation structures in $[217$.

\subsection{Nano-indentation of a thin film}

Indentation of a film of thickness $\sim 30 \mathrm{~nm}$ placed on a rigid substrate is illustrated in this section. The indenter is rectangular in shape and $\sim 18.7 \mathrm{~A}$ wide. The indenter as well as the film are considered to be infinite in the out of plane $X$ direction, thus a plane strain condition exists
in the $Y-Z$ plane (Fig. 6). Indentation direction is $Z$.
Homogeneous Dirichlet boundary condition in $Z$ is
imposed on the bottom face of the film that rests on the
substrate. Homogeneous Dirichlet boundary condition in
$\boldsymbol{Y}$ is imposed on the left and right face of the continutm
problem domain. Indenter load is anplied quasi-statically through Dirichlet boundary condition by moving the

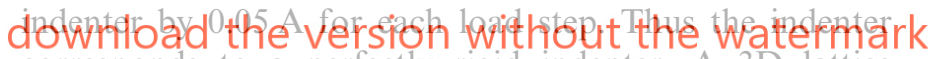
corresponds to a perfectly rigid indenter. A 3D lattice structure is maintained by imposing periodic boundary conditions in $X$ direction for the atomistic model. Crystallographic orientation chosen for the lattice (shown in the left picture of Fig. 6) is such that the dislocations generated

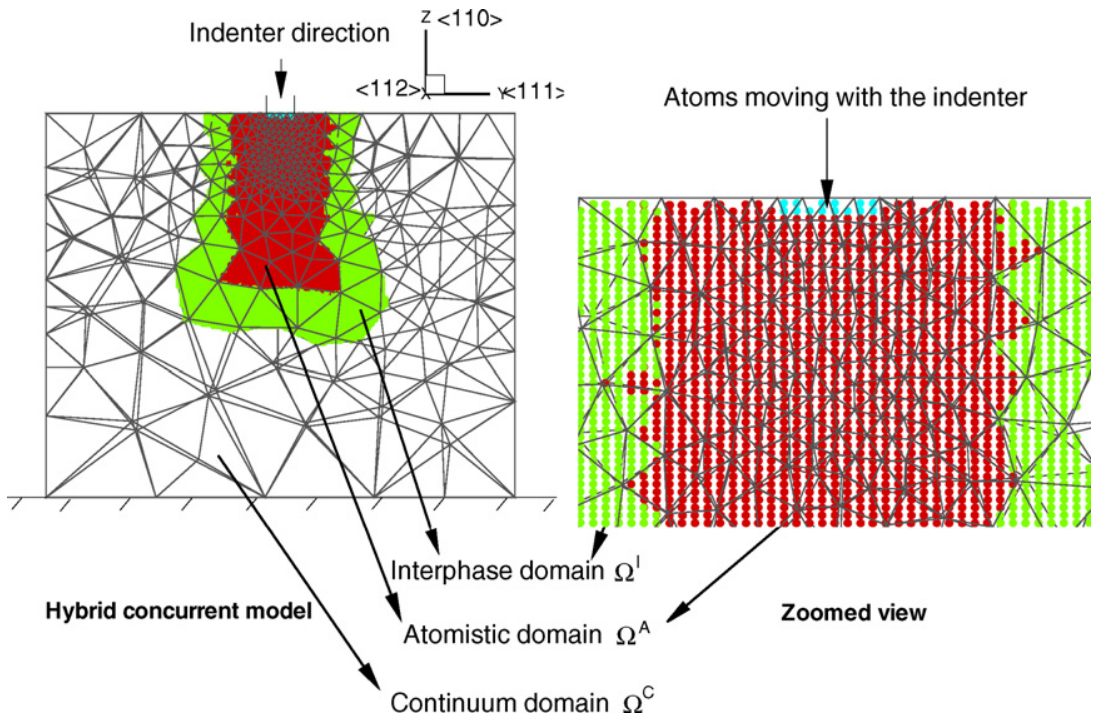

Fig. 6. Hybrid atomistic-continuum concurrent model for nano-indentation. 


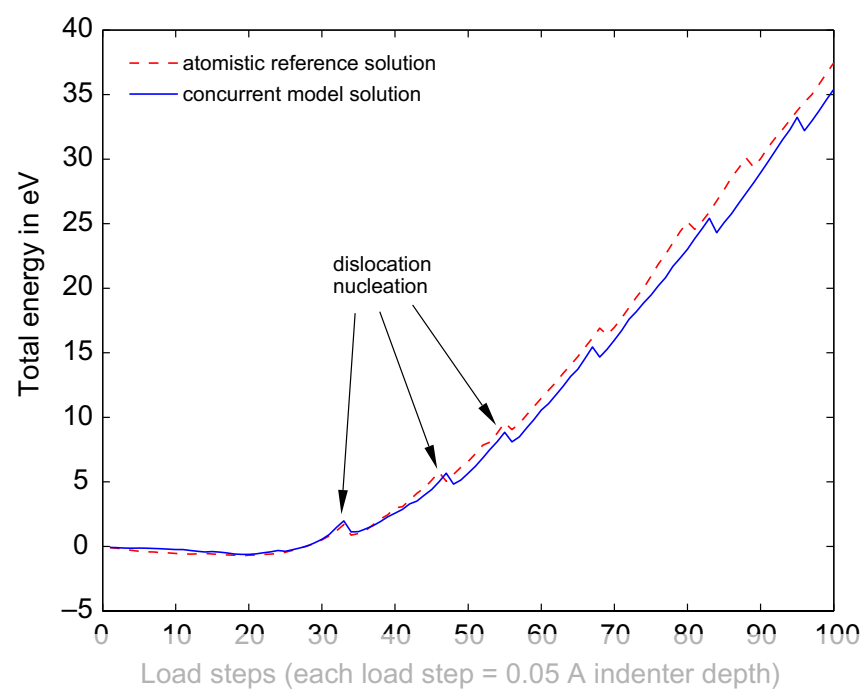

Fig. 7. Energy comparison between atomistic reference solution and the concurrent model solution.

from the corners of the indenter move straight down into the material. Fig. 6 shows a concurrent model for the problem

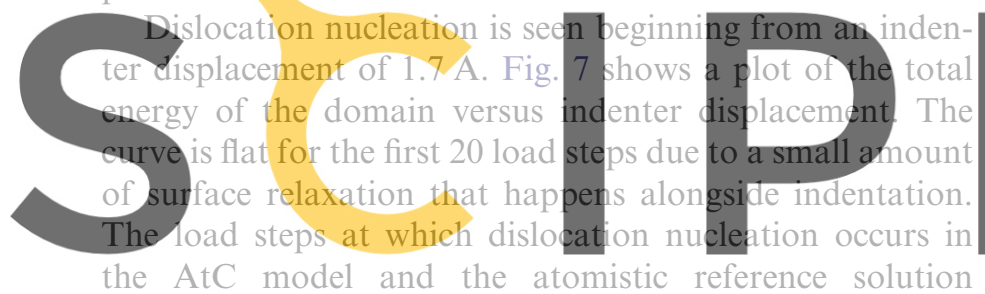

Regre the same except fpr the last two. The load steps at which

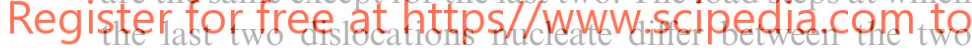
models because of the constraining effect of the finite atomistic region in $\mathrm{AtC}$ on defect nucleation. Thus the AtC model solution agrees well with the atomistic reference solu- tion. Results obtained here also qualitatively agree with the nano-indentation test results presented in [31] although the exact numbers in terms of the indenter displacement at the first dislocation nucleation do not. This is due to difference in indenter size and inter-atomic potential.

The AtC model (Fig. 6) consists of 58,359 total degrees of freedom to solve for. 58,005 are the atomistic degrees of freedom and 354 are the continuum degrees of freedom. Thus the atomistic degrees of freedom dominate the calculations. The $L 2$ norm of the residuals of Eq. (35) was of the order of $10^{-5}$ per degree of freedom at the end of each load step. The atomistic model of the reference solution consists of 173,880 atomistic degrees of freedom.

\subsection{Nano-void subiected to hvdrostatic tension}

A void of size $\sim 50 \mathrm{~A}$ in diameter at the center of a cube of side $\sim 500 \mathrm{~A}$ is subjected to hydrostatic tension. The load is applied quasi-statically in small increments $(0.375 \mathrm{~A})$ through Dirichlet boundary conditions imposed on the outer faces of the cube. Problem simulation consists of solving the problem at each incremental load step. The simulation of a nano-void subjected to hydrostatic tension has

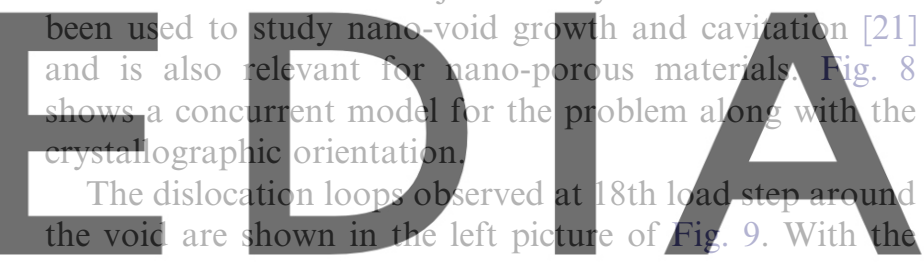
increase in load, dislocation loops grow and quickly react to form Lomer-Cottrell junctions [17]. These junctions downlo a d the versipn nithout the waternark shown in the right portion of Fig. 9, which plots the atoms along the edges of the tetrahedra. Results of the fully atomistic simulation at the corresponding load steps are

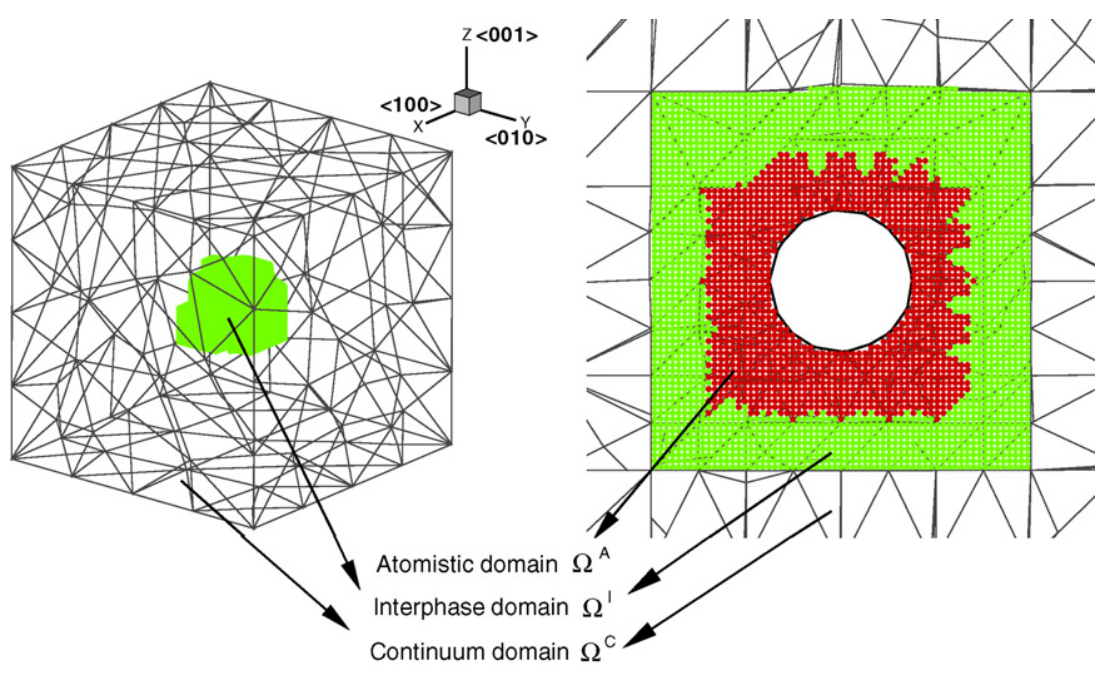

Hybrid concurrent model

Zoomed mid-section view of

the concurrent model

Fig. 8. Hybrid atomistic-continuum concurrent model for Void. 


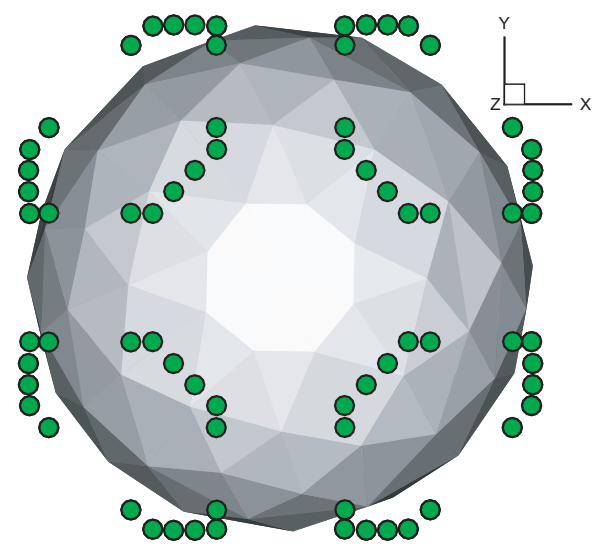

Load step 18

Dislocation loops nucleated from void surface

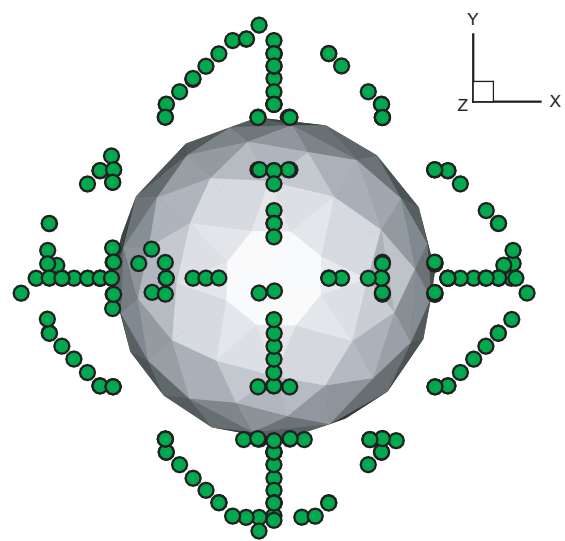

Load step 20

Stacking-fault tetrahera

Fig. 9. Dislocations in the Void problem-concurrent model solution.

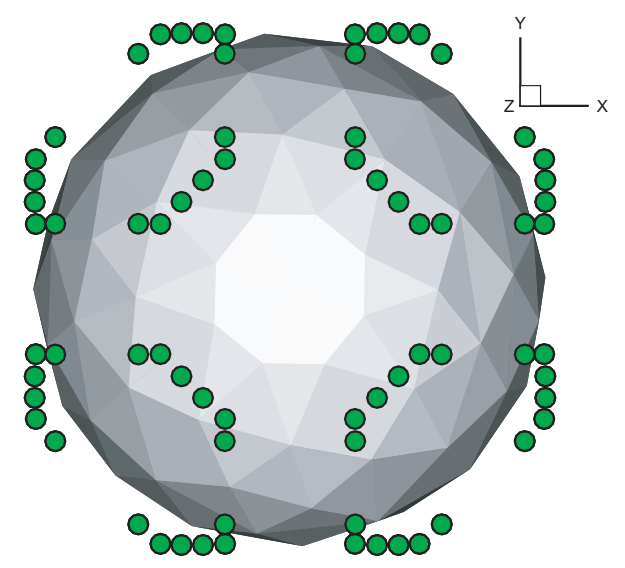

Load step 18

Dislocation loops nucleated from void surface

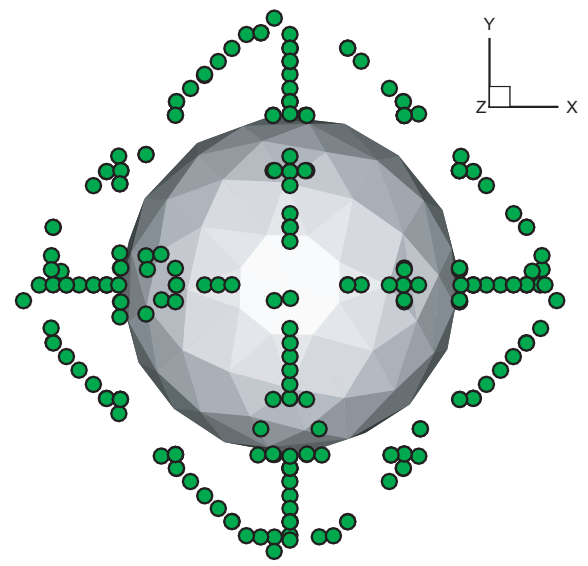

Load step 20

Stacking-fault tetrahedra

Fig. 10. Dislocations in the Void problem-atomistic reference solution.

shown in Fig. 10. The symmetry of the resulting dislocation configuration is due to the crystal symmetry. Results obtained are qualitatively comparable with that presented in [21].

Fig. 11 shows a comparison of the energy plots between the fully atomistic reference solution and the concurrent model (Fig. 8) solution. The energy of the atoms within the inter-atomic cutoff distance from a free surface is subtracted from the model energy to eliminate the energy fluctuations due to surface relaxation. The linear elastic strain energy of the model is also subtracted from the total energy of the model so that the fluctuation in the energy due to nucleation and growth of dislocation loops is clearly distinguishable. Different events noticed during the simulation are also marked in Fig. 11. Stacking fault tetrahedra shown in the right side of Figs. 9 and 10 occur between the load steps 20 and 21 with an associated drop in the energy as shown in Fig. 11.

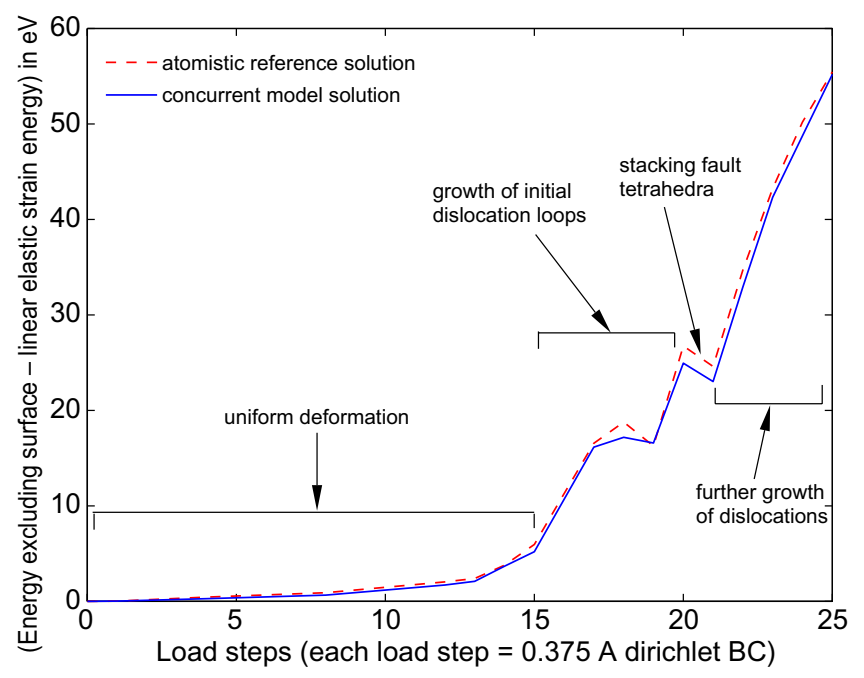

Fig. 11. Comparison of energy between a fully atomistic reference solution and the concurrent model for successive load steps. 
The AtC model (Fig. 8) consists of 331,801 total degrees of freedom. 328,494 are the atomistic degrees of freedom and 3307 are the continuum degrees of freedom. Once again the atomistic degrees of freedom constitute a majority of calculations. The $L 2$ norm of the residuals of Eq. (35) was of the order of $10^{-6}$ per degree of freedom. The atomistic simulation for a reference solution consists of $1,316,412$ degrees of freedom.

\section{Closing remarks}

A concurrent atomistic to continuum (AtC) coupling method is formulated based on a blending of the continuum stress and the atomistic force in the equilibrium equation. The problem domain is decomposed into a continuum sub-domain, an atomistic sub-domain and an overlap interphase sub-domain with a blended atomistic-continuum description. Three different blend functions are considered. Compatibility between the atomistic solution and the continuum solution is imposed within the interphase in a weak sense. Patch test results verified the problem formulation and its implementation. A nano-indentation problem and a nano-void subjected to hydrostatic tension are solved by the AtC method and the results are compared with the results of fully atomistic simulations. Further investigation of the blend functions and weak compatibility constraints is an ongoing work. The AtC method is the basis of an automated adaptive concurrent multiscale procedure currently under development.

\section{Acknowledgments}

We gratefully acknowledge the support of DOE program "A Mathematical Analysis of Atomistic to Continuum Coupling Methods" DE-FG01-05ER05-16 for this work. We also acknowledge NSF for "NIRT: Modeling and Simulation Framework at the Nanoscale: Application to Process Simulation, Nanodevices, and Nanostructured Composites" NSF Grant 0303902 and "Multiscale Systems Engineering for Nanocomposites" NSF Grant 0310596. We also acknowledge useful discussions we had with Pavel Bochev and Richard Lehoucq of the Computational Mathematics and Algorithms group at Sandia National Laboratories, Albuquerque, NM. Santiago Badia acknowledges the support of the European Community through the Marie Curie Contract NanoSim (MOIF-CT-2006-039522).

\section{References}

[1] $<$ http://www.ud.infn.it/ ercolessi/potentials $>$.

[2] Farid F. Abraham, Jeremy Q. Broughton, Noam Bernstein, Efthimios Kaxiras, Spanning the length scales in dynamic simulation, Comput. Phys. 12 (6) (1998) 538.

[3] S. Badia, P. Bochev, J. Fish, M. Gunzburger, R. Lehoucq, M. Nuggehally, M.L. Parks, A force-based blending model for atomisticto-continuum coupling, Int. J. Multiscale Comput. Engrg., submitted for publication.
[4] Ted Belytschko, Wing Kam Liu, Brian Moran, Nonlinear Finite Elements for Continua and Structures, John Wiley \& Sons, Ltd., 2000.

[5] Ted Belytschko, S.P. Xiao, Coupling methods for continuum model with molecular model, Int. J. Multiscale Comput. Engrg. 1 (1) (2003) 115.

[6] Jeremy Q. Broughton, Farid F. Abraham, Noam Bernstein, Efthimios Kaxiras, Concurrent coupling of length scales: methodology and application, Phys. Rev. B 60 (4) (1999) 2391.

[7] X.C. Cai, W.D. Gropp, D.E. Keyes, A comparison of some domain decomposition and ilu preconditioned iterative methods for nonsymmetric elliptic problems, Numer. Linear Algebr. Appl. 1 (1994) 477.

[8] Willian A. Curtin, Ronald E. Miller, Atomistic/continuum coupling in computational material science, Model. Simul. Mater. Sci. Engrg. 11 (2003) 33.

[9] Dibyendu K. Datta, Catalin R. Picu, Mark S. Shephard, Composite grid atomistic continuum method: an adaptive approach to bridge continuum with atomistic analysis, Int. J. Multiscale Comput. Engrg. 2 (2004) 401.

[10] Murray S. Daw, M.I. Baskes, Embedded-atom method: derivation and application to impurities, surfaces, and other defects in metals, Phys. Rev. B 29 (12) (1984) 6443.

[11] Hashmi Ben Dhia, Guillaume Rateau, The arlequin method as a flexible engineering design tool, Int. J. Numer. Methods Engrg. 62 (2005) 1442.

[12] Laurent M. Dupuy, Ellad B. Tadmor, Ronald E. Miller, Rob Phillips, Finite-temperature quasicontinuum: molecular dynamics without all the atoms, Phys. Rev. Lett. 95 (060202) (2005) 1.

[13] F. Ercolessi, J.B. Adams, Interatomic potentials from first-principles calculations: the force-matching method, Europhys. Lett. 26 (1994) 583.

[14] Gerald Farin, Curves and Surfaces for Computer Aided Geometric Design: A Practical Guide, Academic Press, Inc., 1988.

[15] Jacob Fish, Wen Chen, Discrete-to-continuum bridging based on multigrid principles, Comput. Methods Appl. Mech. Engrg. 193 (2004) 1693.

[16] Thomas J.R. Hughes, The Finite Element Method: Linear Static and Dynamic Finite Element Analysis, Dover, 2000.

[17] Derek Hull, David J. Bacon, Introduction to Dislocations, Butterworth Heinemann, 2001.

[18] Cynthia L. Kelchner, S.J. Plimpton, J.C. Hamilton, Dislocation nucleation and defect structure during surface indentation, Phys. Rev. B 58 (17) (1998) 11085.

[19] S. Kohlhoff, P. Gumbsch, H.F. Fischmeister, Crack propagation in b.c.c. crystals studied with a combined finite-element and atomistic model, Philos. Mag. A 64 (4) (1991) 851.

[20] B.Q. Luan, S. Hyun, J.F. Molinari, N. Bernstein, Mark O. Robbins, Multiscale modeling of two-dimensional contacts, Phys. Rev. E 74 (046710) (2006) 1.

[21] Jaime Marian, Jaroslaw Knap, Michael Ortiz, Nanovoid cavitation by dislocation emission in aluminum, Phys. Rev. Lett. 93 (165503) (2004) 1.

[22] Ronald E. Miller, Ellad B. Tadmor, The quasicontinuum method: overview, applications and current directions, J. Comput.-Aided Mater. Des. 9 (3) (2002) 203.

[23] M. Mullins, M.A. Dokainish, Crack propagation in b.c.c. crystals studied with a combined finite-element and atomistic model, Philos. Mag. A 46 (4) (1982) 771.

[24] Harold S. Park, Eduard G. Karpov, Wing Kam Liu, A temperature equation for coupled atomistic/continuum simulations, Comput. Methods Appl. Mech. Engrg. 193 (2004) 1713.

[25] Catalin R. Picu, On the functional form of non-local elasticity kernels, J. Mech. Phys. Solids 50 (2002) 1923.

[26] R. Qiao Narayana, R. Aluru, Atomistic simulation of kc1 transport in charged silicon nanochannels: interfacial effects, Colloids Surf. A: Physiochem. Engrg. Aspects 267 (2005) 103. 
[27] Robert E. Rudd, Coarse-grained molecular dynamics for computer modeling of nanomechanical systems, Int. J. Multiscale Comput. Engrg. 2 (2) (2004) 203.

[28] Robert E. Rudd, Jeremy Q. Broughton, Coarse-grained molecular dynamics and the atomistic limit of finite elements, Phys. Rev. B 58 (10) (1998) 5893.

[29] L.E. Shilkrot, William A. Curtin, Ronald E. Miller, A coupled atomistic/continuum model of defects in solids, J. Mech. Phys. Solids 50 (2002) 2085.

[30] L.E. Shilkrot, Ronald E. Miller, William A. Curtin, Multiscale plasticity modeling: coupled atomistics and discrete dislocation mechanics, J. Mech. Phys. Solids 52 (2004) 755.

[31] E.B. Tadmor, R. Miller, R. Phillips, Nanoindentation and incipient plasticity, J. Mater. Res. 14 (6) (1999) 2233.
[32] Ellad B. Tadmor, Michael Ortiz, Rob Phillips, Quasicontinuum analysis of defects in solids, Philos. Mag. A 73 (6) (1996) 1529.

[33] Gregory J. Wagner, Wing Kam Liu, Coupling of atomistic and continuum simulations using a bridging scale decomposition, J. Comput. Phys. 190 (2003) 249.

[34] S.P. Xiao, Ted Belytschko, A bridging domain method for coupling continua with molecular dynamics, Comput. Methods Appl. Mech. Engrg. 193 (2004) 1645.

[35] Min Zhou, A new look at the atomic level virial stress: on continuummolecular system equivalence, Proc. Royal Soc. Lond. Ser. A 459 (2003) 2347.

[36] O.C. Zienkiewicz, The Finite Element Method, third ed., Tata McGraw-Hill, 2003. 